\title{
CRESCIMENTO EM DIÂMETRO DO TRONCO DAS ÁRVORES DE Eucalyptus grandis W. HILL. EX. MAIDEN E RELAÇÃO COM AS VARIÁVEIS CLIMÁTICAS E FERTILIZAÇÃO MINERAL ${ }^{1}$
}

\author{
Carlos Roberto Sette $\mathrm{Jr}^{2}$, Mario Tomazello Filho ${ }^{3}$, Carlos Tadeu dos Santos Dias ${ }^{3}$ e Jean Paul Laclau ${ }^{4}$
}

\begin{abstract}
RESUMO - Este trabalho teve como objetivo avaliar o incremento em diâmetro do tronco de árvores de Eucalyptus grandis por 24 meses e sua relação com as variáveis climáticas e fertilização mineral. As árvores foram plantadas no espaçamento de 3 x 2 m e fertilizadas com potássio e sódio (plantio, 6 e 12 meses). Foram selecionadas 20 árvores de eucalipto por tratamento, de acordo com a distribuição de área basal do povoamento e instaladas faixas dendrométricas na altura do DAP. Os resultados indicaram efeito da sazonalidade climática no incremento em diâmetro do tronco das árvores, com períodos de máximo e de mínimo crescimento e uma defasagem (lag) de 28 dias, em função da resposta da atividade cambial às variações climáticas. A aplicação de potássio em relação ao sódio e controle promoveu maior taxa de incremento acumulado do tronco das árvores, com valores de 4,14; 3,28; e 3,08 cm, respectivamente.
\end{abstract}

Palavras-chave: Dendrômetro, Variáveis climáticas, Fertilização e Eucalipto.

\section{GROWTH IN DIAMETER OF Eucalyptus grandis W. HILL. EX MAIDEN TREES AND RELATIONSHIP WITH CLIMATIC VARIABLES AND MINERAL FERTILIZATION}

\begin{abstract}
The present work had the objective to evaluate the increment in diameter of Eucalyptus grandis trees for 24 months and its relationship with climatic variables and mineral fertilization. The trees were planted at spacing of $3 \times 2 \mathrm{~m}$ and fertilized with potassium and sodium (planting, 6 and 12 months). Twenty eucalypt trees were selected by treatment according with the distribution of basal area of the plantation and dendrometer bands were fixed at DBH. The results showed a clear effect of climate seasonality on the increment of eucalypt trees, with periods of maximum and minimum growth and a delay period (lag) of 28 days, due to the response of cambial activity to climate variations. The application of potassium in relationship of sodium and control promoted greater rate of increment growth of eucalypt trees, with values of $4.14 ; 3.28$ and $3.08 \mathrm{~cm}$, respectively.
\end{abstract}

Keywords: Dendrometer, Climatic variables, Fertilization and Eucalypt.

\footnotetext{
${ }^{1}$ Recebido em 26.11.2008 e aceito para publicação em 25.08.2010.

${ }^{2}$ Universidade Federal de São Carlos, UFSCAR, Brasil. E-mail: <csette@ufscar.br>.

${ }^{3}$ Universidade de São Paulo, USP, Brasil. E-mail: <mtomazel@esalq.usp.br>.

${ }^{4}$ Centre de recherche agronomique pour le développement, CIRAD, França.
} 


\section{INTRODUÇÃO}

O crescimento em comprimento e em diâmetro das árvores é resultado da atividade dos meristemas apical e cambial, respectivamente (HUSCH et al., 1982). Dessa forma, o crescimento é influenciado pela genética da espécie e, também, pelas condições do ambiente que compreendem, basicamente, os fatores climáticos, edáficos, topográficos e de competição (LAMPRECHT, 1990).

O monitoramento contínuo do crescimento radial do tronco é fundamental para entender a reação das árvores aos estímulos e variações das condições climáticas, como a temperatura, precipitação e déficit de pressão de vapor, bem como os decorrentes da aplicação de fertilizantes minerais e orgânicos. O uso de dendrômetros permanentes tem-se mostrado opção eficiente na medição contínua do crescimento em diâmetro do tronco das árvores de florestas tropicais e temperadas, avaliando pequenas mudanças no seu crescimento em intervalos de tempo reduzidos, além da alta precisão e baixo custo (MARIAUX, 1967, 1969 e 1970; DÉTIENNE et al., 1988; BOTOSSO e TOMAZELLO FILHO, 2001). As árvores de eucalipto em plantações florestais, monitoradas com dendrômetros, apresentam sazonalidade do crescimento em diâmetro do tronco influenciada pelas condições climáticas, como a precipitação, temperatura, déficit de pressão de vapor etc. (VALENZIANO e SCARAMUZZI, 1967; GREEN, 1969; MARIEN e THIBOUT, 1980; DOWNES et al., 1999; WIMMER et al., 2002; LACLAU et al., 2005; DREW et al., 2008). Para Fahn et al. (1981), a periodicidade da atividade do câmbio parece ser decorrente de alterações na temperatura, comprimento do dia e precipitação pluviométrica, com os fatores endógenos inerentes a cada espécie controlando o seu ritmo de crescimento. Da mesma forma, o efeito significativo da fertilização mineral no crescimento do eucalipto é reconhecido há algumas décadas, a exemplo de Mello (1968), que demonstrou a diferença de produção volumétrica de $33 \mathrm{~m}^{3} /$ ha das árvores de E. saligna adubadas e não adubadas, aos 2 anos de idade, em solos pobres e ácidos de cerrado. O incremento de volume do tronco de Eucalyptus saligna e de E. grandis com adubação potássica variou de 67 a 118\% no Cerrado do Estado de Minas Gerais (BARROS et al., 1981 apud SILVEIRA e MALAVOLTA, 2003) e do Estado de São Paulo (GAVA, 1997), respectivamente.
Este trabalho teve como objetivo avaliar o incremento em diâmetro do tronco das árvores de Eucalyptus grandis por 24 meses, utilizando faixas dendrométricas e determinando sua relação com as variáveis climáticas e fertilização mineral.

\section{MATERIAL E MÉTODOS}

\subsection{Local}

O experimento foi instalado na Estação Experimental de Itatinga, SP, do Departamento de Ciências Florestais da ESALQ/USP, localizada a $23^{\circ} 10^{\prime} \mathrm{S}$ e $48^{\circ} 40^{\prime} \mathrm{W}$, com $857 \mathrm{~m}$ de altitude. $\mathrm{O}$ clima da região é caracterizado como mesotérmico úmido (Cwa), segundo Köeppen, com precipitação média anual de 1.200 mm e temperatura média de $16,2^{\circ} \mathrm{C}$ e $28,6^{\circ} \mathrm{C}$ nos meses mais frio e mais quente, respectivamente (Tabela 1 ).

Osolo édo tipo Latossolo Vermelho-Amarelo distrófico de textura média( $200 \mathrm{~g} \mathrm{~kg}^{-1}$ de argila), cuja litologiaé composta por arenito, formação Marília, pertencendo ao Grupo Bauru. Os resultados da análise do solo na fase da implantação do experimento encontram-se na Tabela 2.

Tabela 1 -Dados mensais de precipitação (Prec.), temperatura média (Temp.) e déficit de pressão de vapor (DPV) da área experimental.

Table 1 -Monthly data of precipitation (Prec.), mean temperature (Temp) and vapor pressure deficit (DPV) of the experimental area.

\begin{tabular}{lcccc}
\hline Ano & Mês & Prec. $(\mathrm{mm})$ & Temp.(C) & DPV(kPA) \\
\hline 2006 & fev & 128,0 & 27,7 & 1,3 \\
& mar & 203,5 & 26,9 & 1,0 \\
& abr & 107,0 & 23,6 & 0,9 \\
& mai & 2,2 & 20,0 & 0,9 \\
& jun & 0,4 & 19,8 & 1,0 \\
& jul & 47,6 & 21,2 & 1,0 \\
& ago & 26,1 & 19,9 & 1,2 \\
& set & 91,8 & 22,5 & 1,0 \\
& out & 80,9 & 25,4 & 1,0 \\
& nov & 95,5 & 25,5 & 1,0 \\
& dez & 177,7 & 26,6 & 1,1 \\
\hline 2007 & jan & 422,4 & 25,7 & 1,0 \\
& fev & 29,3 & 26,4 & 1,3 \\
& mar & 186,5 & 26,9 & 1,3 \\
& abr & 26,7 & 25,8 & 1,3 \\
& mai & 61,0 & 22,4 & 1,0 \\
& jun & 25,2 & 20,6 & 0,9 \\
& jul & 56,3 & 16,2 & 1,1 \\
& ago & 124,8 & 19,2 & 1,1 \\
& set & 2,5 & 24,0 & 1,4 \\
& out & 10,5 & 26,8 & 1,4 \\
& nov & 201,5 & 23,8 & 1,0 \\
& dez & 177,3 & 24,4 & 1,1 \\
\hline jan & 243,7 & 28,6 & 1,1 \\
& fev & 130,4 & 25,3 & 1,0 \\
\hline
\end{tabular}


Tabela 2 - Atributos químicos do solo da área experimental. Table 2 - Soil chemical attributes of the experimental area.

\begin{tabular}{|c|c|c|c|c|c|c|c|c|c|}
\hline \multirow{2}{*}{$\begin{array}{c}\text { Profundidade } \\
\text { M }\end{array}$} & \multicolumn{2}{|c|}{$\mathrm{pH}$} & \multirow[t]{2}{*}{$\mathrm{K}$} & \multirow[t]{2}{*}{$\mathrm{Ca}$} & \multirow[t]{2}{*}{$\mathrm{Mg}$} & \multirow{2}{*}{$\frac{\mathrm{Ma}}{\mathrm{mmol}_{\mathrm{c}} \mathrm{Kg}^{-1}}$} & \multirow[t]{2}{*}{$\mathrm{H}+\mathrm{AI}$} & \multirow[t]{2}{*}{$\mathrm{SB}$} & \multirow[t]{2}{*}{ CTC } \\
\hline & água & $\mathrm{KCI}$ & & & & & & & \\
\hline $0-0,05$ & 4,6 & 4,0 & 0,1246 & 0,2180 & 0,3115 & 0,0000 & 23,54 & 1,40 & 24,94 \\
\hline $0,05-0,15$ & 5,3 & 4,4 & 0,0467 & 0,0081 & 0,1003 & 0,0000 & 11,66 & 0,30 & 11,95 \\
\hline $0,15-0,50$ & 5,5 & 4,7 & 0,0286 & 0,0052 & 0,0316 & 0,0000 & 8,80 & 0,99 & 8,89 \\
\hline $0,50-1,0$ & 5,5 & 4,6 & 0,0161 & 0,0000 & 0,0124 & 0,0000 & 6,49 & 0,04 & 6,53 \\
\hline $1,0-2,0$ & 5,7 & 5,2 & 0,0305 & 0,0153 & 0,0153 & 0,0034 & 3,94 & 0,12 & 4,06 \\
\hline $2,0-3,0$ & 6,0 & 5,3 & 0,0133 & 0,0007 & 0,0103 & 0,0159 & 0,80 & 0,08 & 0,87 \\
\hline $3,04,0$ & 5,7 & 5,3 & 0,0393 & 0,363 & 0,0375 & 0,0042 & 0,28 & 0,16 & 0,44 \\
\hline $4,0-5,0$ & 5,9 & 5,5 & 0,0298 & 0,0261 & 0,0539 & 0,0000 & 0,04 & 0,16 & 0,20 \\
\hline $5,0-6,0$ & 5,8 & 5,6 & 0,0287 & 0,0095 & 0,0164 & 0,0072 & 0,13 & 0,11 & 0,24 \\
\hline
\end{tabular}

Fonte: MAQUÈRE, 2006 apud ALMEIDA, 2009.

Source: MAQUÈRE, 2006 apud ALMEIDA, 2009.

\subsection{Delineamento experimental}

O incremento em diâmetro do tronco das árvores de Eucalyptus grandis foi avaliado em experimento implantado em abril/2004 ( $1^{\text {a }}$ rotação), no espaçamento de $3 \times 2 \mathrm{~m}$, delineamento experimental em quatro blocos casualizados com três tratamentos $\left(\mathbf{T}_{1}\right.$ : controle; $\mathbf{T}_{2}$ : aplicação de 116 kg/ha de K na forma de KCl no plantio, 6 e 12 meses; e $\mathbf{T}_{3}$ : aplicação de 68,5 Kg/ha de $\mathrm{Na}$ na forma de $\mathrm{NaCl}$ no plantio, 6 e 12 meses). Todos os tratamentos receberam $80 \mathrm{kgha}^{-1} \mathrm{deP}_{2} \mathrm{O}_{5}, 120 \mathrm{kgha}^{-1} \mathrm{deN}\left(\mathrm{NH}_{4}(\mathrm{SO} 4)_{2}\right)$, $45 \mathrm{~kg} \mathrm{ha}^{-1}$ de micronutrientes (FTE BR-12) e $45 \mathrm{~kg} /$ ha $^{-1}$ deboro(Borogran)(LACLAU etal., 2007). Oexperimento foi desenvolvido no âmbito do projeto "Resposta de árvores de Eucalyptus grandis à adubação potássica e à substituição do potássio por sódio”.

\subsection{Seleção das árvores}

Para a avaliação do incremento em diâmetro do tronco, foram selecionadas 20 árvores de eucalipto com 21 meses, por tratamento, de acordo com a distribuição de área basal, totalizando 60 árvores. O monitoramento do incremento em diâmetro do tronco foi feito por um período de 24 meses (fevereiro de 2006 a fevereiro de 2008).

\subsection{Incremento em diâmetro do tronco das árvores de eucalipto}

Foram instaladas faixas dendrométricas permanentes a 1,30 m de altura no tronco das árvores de eucalipto (Figura 1A), confeccionadas de acordo com a metodologia descrita por Botosso e Tomazello Filho
(2001): fitas de aço inoxidável de 12,7 x 0,15 mm (largura e espessura), com escala em mm e nônio com precisão de leitura de 0,2 mm, mantidas sob tração por uma mola de aço inoxidável de 100 x 8 mm (comprimento e diâmetro) (Figura $1 \mathrm{AB}$ ) no tronco. Na montagem das faixas dendrométricas, determinou-se a circunferência do tronco das árvores e cortou-se a fita de aço em comprimento referente ao seu valor e com $17 \mathrm{~cm}$ adicionais para sobreposição da escala e do nônio. Posteriormente, as graduações da escala e nônio foram demarcadas na fita de aço com o auxílio de placas de metal de gabarito (Figura 1C), com graduação de $80 \mathrm{~mm}$ da escala de leitura (borda inferior da placa) e 10 divisões do nônio (borda superior da placa).

Na avaliação do incremento em diâmetro do tronco das árvores foi mensurado o deslocamento das escalas sobrepostas das faixas dendrométricas a cada 14 dias, no período da manhã (8-10 h) e em um mesmo percurso. Os dados registrados foram transferidos para planilhas eletrônicas e transformados em gráficos, sendo, então, analisados e interpretados para posterior correlação com as variáveis climáticas e tratamentos de fertilização.

\subsection{Variáveis climáticas}

Os valores das temperaturas média, máxima e mínima $\left({ }^{\circ} \mathrm{C}\right)$, precipitação acumulada $(\mathrm{mm})$ e umidade do solo (\%) no período de janeiro/2006 a fevereiro/2008 foram coletados na Estação Meteorológica da Estação Experimental de Itatinga, SP (800 m da área experimental) e agrupados a cada 14 dias, de modo a coincidir com as leituras das faixas dendrométricas, para posterior correlação com os valores de incremento em diâmetro

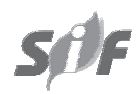

Revista Árvore, Viçosa-MG, v.34, n.6, p.979-990, 2010 


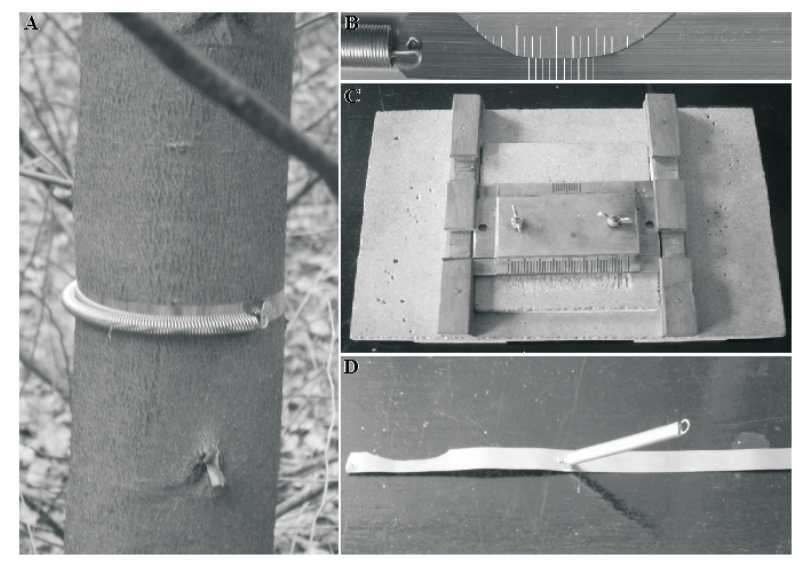

Figura 1 - Confecção e instalação da faixa dendrométrica na árvore: (A) faixa dendrométrica instalada no tronco da árvore, (B) detalhe da sobreposição da escala e do nônio, (C) gabarito (D) detalhe da faixa dendrométrica com escala e mola.

Figure 1 - Preparation and installation of the dendrometer band in the tree: (A) dendrometer band installed in the tree; $(B)$ detail of the scale and vernier; (C) recording (D) detail of the dendrometer band with scale.

do tronco do eucalipto. O déficit de pressão de vapor (DPV) foi determinado pelo método de Penman-Monteith FAO (PM-FAO apud DELGADO, 2007), com a pressão de saturação de vapor (es) calculada pela temperatura média (TM) e a pressão parcial de vapor (ea) pela umidade relativa do ar (UR):

$$
\begin{aligned}
& e s=0,611 e^{17,27 T M / T M+237} \\
& e a=\frac{U R}{100} * e s \\
& D P V=(e s-e a)
\end{aligned}
$$

\subsection{Análises estatísticas}

Nas análises estatísticas foi aplicado o programa SAS, sendo aferidos os “outliers”, heterogeneidade da variância e escala pelo Método de Potência Ótima de Box-Cox para a melhor transformação. A análise de variância (ANOVA) foi aplicada para verificar o efeito dos tratamentos e, quando significativo, o teste de Tukey a 95\% de probabilidade. Na análise dos dados de incremento em diâmetro do tronco das árvores foram realizados os testes de (i) correlação com as variáveis climáticas e (ii) regressão utilizando o procedimento “stepwise”. Como as árvores reagem em crescimento em diâmetro do tronco, após um período de ocorrência das variáveis climáticas foi aplicada a autorregressão para determinar esse período de defasagem (lag). Na definição desse período, consideraram o coeficiente de correlação e a significância (5\% de probabilidade) dos lags na autorregressão.

\section{RESULTADOS E DISCUSSÃO}

As faixas dendrométricas de aço mostraram-se eficientes na avaliação do incremento do tronco, em resposta ao clima e à fertilização, conforme apresentado a seguir:

\subsection{Efeito das variáveis climáticas}

Os incrementos máximos em diâmetro do tronco nos 24 meses de monitoramento ocorreram de fevereiro a maio, setembro, início de outubro e dezembro de 2006; e maio, julho, agosto e novembro de 2007 (Tabela 3; Figura 2A). Com relação a esse período, as taxas de crescimento acumulado em diâmetro do tronco foram de 1,70; 2,44; e 1,83 cm, nos Tratamentos 1, 2 e 3, representando 55, 59 e $56 \%$ do incremento total no período de 24 meses (Tabela 4), com destaque para os meses de março, maio e dezembro de 2006 e novembro de 2007, com valores de 3 a $7 \%$ (Tabela 3).

O período de máximo crescimento do tronco das árvores, à exceção de maio de 2006, pode ser relacionado aos altos níveis de precipitação e temperatura e ao baixo déficit de pressão de vapor (Tabela 1; Figura 4), ao estádio fenológico das árvores (copa formada, folhas maduras com limbo expandido) e à disponibilidade de água no solo (Figura 3) e de horas de luz/dia, com o aumento da taxa de fotossíntese. Nesse período, os hormônios e carboidratos, ao deslocarem-se no sentido descendente do tronco pelo floema, induziram maior taxa de divisão das células cambiais, com o consequente aumento do crescimento do tronco.

A taxa de incremento em diâmetro do tronco das árvores em maio de 2006 (com baixa pluviosidade e redução da temperatura) é resultado da disponibilidade de água da estação de chuvas, armazenada nas camadas mais profundas do solo (Figura 3). Nesse sentido, Valenziano e Scaramuzzi (1967), Downes et al. (1999) e Drew et al. (2008) obtiveram a mesma resposta do crescimento do diâmetro das árvores de eucalipto no período de seca, atribuída à água disponível a vários metros do solo. 
Tabela 3 - Incremento em diâmetro do tronco das árvores de eucalipto entre dois períodos de medição. Table 3 -Increment in diameter of eucalyptus trees between 2 dates of measurements.

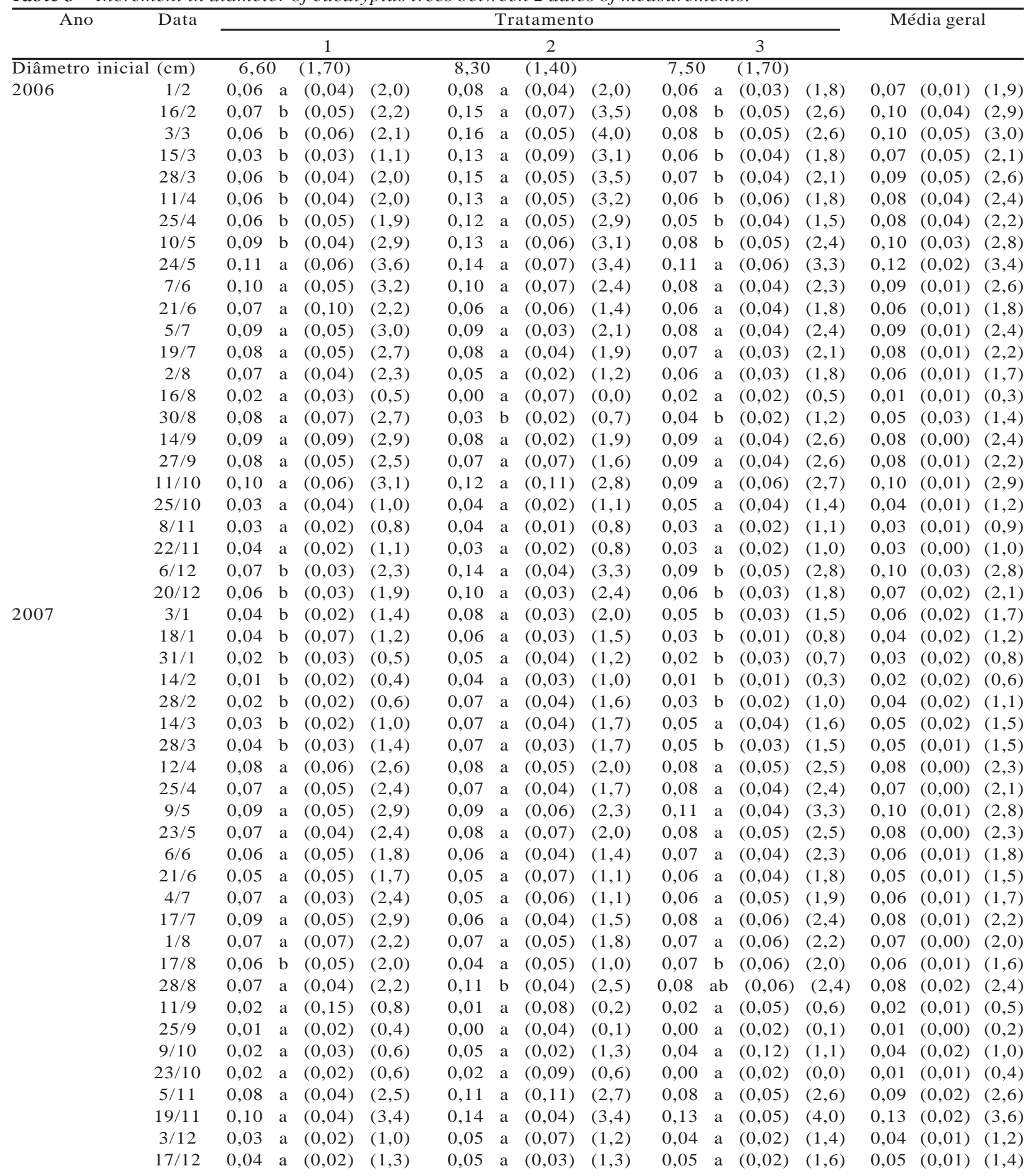


Tabela 3 - Cont.

Table 3 - Cont.

\begin{tabular}{|c|c|c|c|c|c|c|c|c|c|c|c|c|c|c|c|}
\hline \multirow[t]{5}{*}{2008} & $2 / 1$ & 0,03 & $(0,02)$ & $(1,0)$ & 0,05 & $\mathrm{a}$ & $(0,04)$ & $(1,2)$ & 0,03 & $\mathrm{a}$ & $(0,03)$ & $(1,0)$ & 0,04 & $(0,01)$ & $(1,1)$ \\
\hline & $15 / 1$ & $0,04 \quad a$ & $(0,03)$ & $(1,2)$ & 0,05 & $\mathrm{a}$ & $(0,04)$ & $(1,2)$ & 0,04 & $\mathrm{a}$ & $(0,03)$ & $(1,2)$ & 0,04 & $(0,01)$ & $(1,2)$ \\
\hline & $28 / 1$ & 0,04 a & $(0,03)$ & $(1,1)$ & 0,05 & a & $(0,04)$ & $(1,3)$ & 0,05 & a & $(0,04)$ & $(1,6)$ & 0,05 & $(0,01)$ & $(1,4)$ \\
\hline & $12 / 2$ & $0,05 \quad b$ & $(0,04)$ & $(1,5)$ & 0,09 & $\mathrm{a}$ & $(0,05)$ & $(2,1)$ & 0,07 & $a b$ & $(0,04)$ & $(2,2)$ & 0,07 & $(0,02)$ & $(1,9)$ \\
\hline & $26 / 2$ & $0,03 \mathrm{~b}$ & $(0,02)$ & $(0,8)$ & 0,06 & $\mathrm{a}$ & $(0,04)$ & $(1,5)$ & 0,05 & $\mathrm{a}$ & $(0,03)$ & $(1,5)$ & 0,05 & $(0,02)$ & $(1,3)$ \\
\hline Diâmetro final & $(\mathrm{cm})$ & 9,68 & $(2,36)$ & & 12,44 & & $(2,66)$ & & 10,7 & & $(2,18)$ & & & & \\
\hline
\end{tabular}

Médias seguidas de desvio-padrão e porcentagem em relação ao incremento acumulado durante os 24 meses de monitoramento (IA); em uma mesma linha, médias seguidas de pelo menos uma letra igual não diferem significativamente $(\mathrm{p}>0,05)$.

Means followed by standard deviation and percentage relative to the 24 months of monitoring (IA) in the same row, means followed by at least an equal letter do not differ significantly $(p>0.05)$.

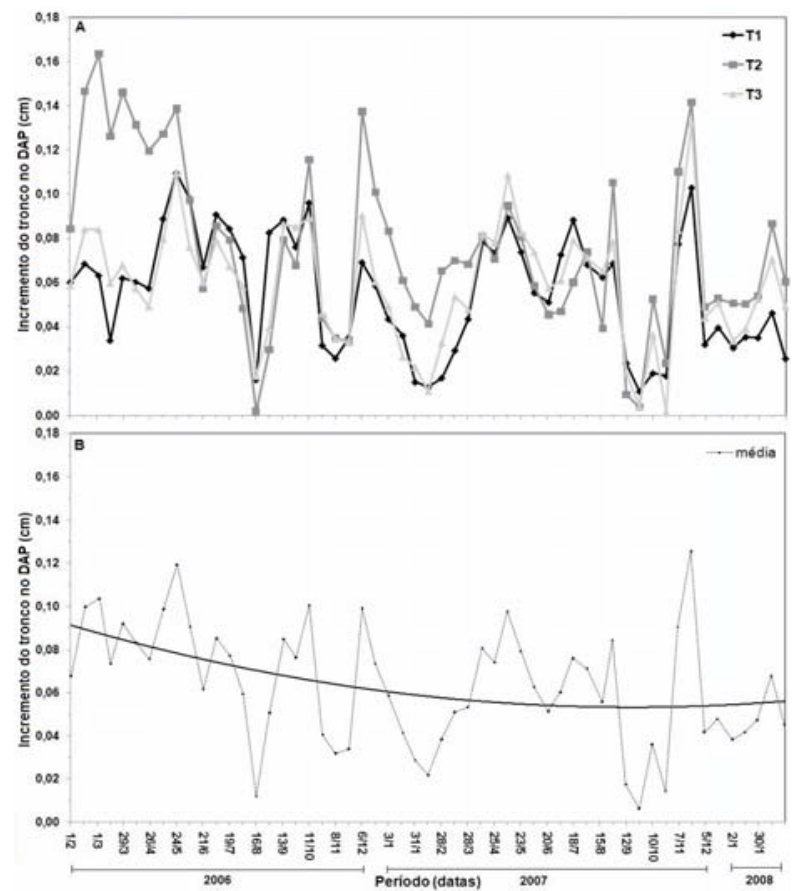

Figura 2 - Incremento do tronco das árvores de eucalipto nos três tratamentos (A) e curva de tendência média dos tratamentos (B).

Figure 2-Increment of eucalyptus trees in the three treatments (A) and average trend line of treatments (B).

Os incrementos mínimos em diâmetro do tronco das árvores nos 24 meses ocorreram em agosto, final de outubro e novembro de 2006 e em janeiro, fevereiro, setembro e outubro de 2007 (Tabela 3; Figura 2A). Com relação a esse período, as taxas de crescimento acumuladas em diâmetro do tronco foram de 0,30; 0,50; e $0,33 \mathrm{~cm}$, nos tratamentos 1 , 2 e 3 , respectivamente, representando apenas $10-12 \%$ do incremento total (Tabela 4).

Revista Árvore, Viçosa-MG, v.34, n.6, p.979-990, 2010
Esse período de crescimento mínimo em diâmetro do tronco é caracterizado por níveis limitantes de precipitação, exceto de outubro-novembro/2006 e janeiro/ 2007, com 68,4 mm (Tabela 1; Figura 4). Resposta similar em crescimento do tronco foi verificada em árvores de várias espécies de eucalipto em estudos realizados por Valenziano e Scaramuzzi (1967), Green (1969), Marien e Thibout (1980), Poole (1986) e Wimmer et al. (2002ab). Em outubro-novembro/2006, com precipitação de 176 mm e temperatura média de $25,4^{\circ} \mathrm{C}$, o crescimento em diâmetro do tronco foi característico do período de mínimo crescimento. Essa redução do crescimento em diâmetro do tronco das árvores foi observada na República do Congo por Laclau et al. (2005), em árvores de eucalipto, dois meses após o início das primeiras chuvas da estação, que alocam os nutrientes e carboidratos para os meristemas vegetativos e para a formação de raízes finas, em detrimento do meristema cambial.

A redução do incremento em diâmetro do tronco das árvores em janeiro de 2007, apesar do alto nível de precipitação (422 mm), deve ser parcialmente atribuída

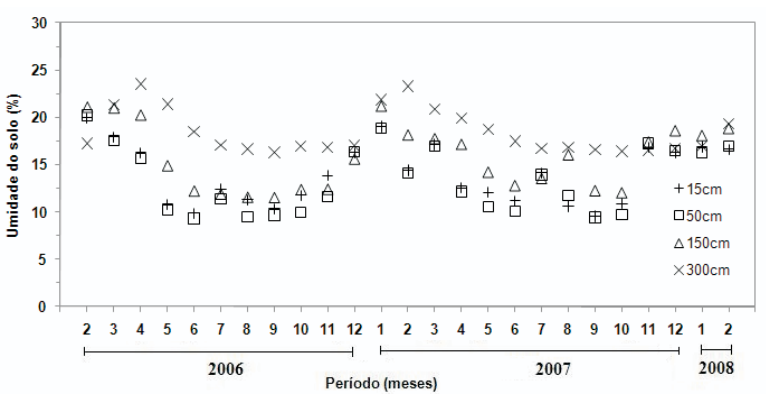

Figura 3 - Umidade do solo da área experimental: avaliação mensal a diferentes profundidades.

Figure 3-Soil moisture of the experimental area: monthly evaluation in different depths. 
Tabela 4 - Incremento em diâmetro do tronco das árvores de eucalipto.

Table 4 - Increment in diameter of eucalyptus trees.

\begin{tabular}{cccc}
\hline Parâmetro $(\mathrm{cm})$ & \multicolumn{3}{c}{ Tratamento } \\
\cline { 2 - 4 } & 1 & 2 & 3 \\
\hline IA1 & $3,08(0,02)(100)$ & $4,14(0,04)(100)$ & $3,28(0,03)(100)$ \\
IAmáx & $1,70(0,02)(55)$ & $2,44(0,03)(59)$ & $1,83(0,02)(56)$ \\
IAmin $^{3}$ & $0,30(0,01)(10)$ & $0,50(0,02)(12)$ & $0,33(0,02)(10)$ \\
IMM $^{4}$ & $0,06(0,02)$ & $0,08(0,04)$ & $0,06(0,03)$ \\
IMAmáx $^{5}$ & $0,07(0,03)$ & $0,11(0,03)$ & $0,08(0,02)$ \\
IMAmin $^{6}$ & $0,02(0,01)$ & $0,04(0,02)$ & $0,03(0,02)$ \\
\hline
\end{tabular}

Médias seguidas de desvio-padrão e porcentagem dos incrementos acumulados máximo (IAmáx) e mínimo (IAmín) em relação ao incremento acumulado no período (IA); incremento em diâmetro do tronco acumulado no período: (i) total ${ }^{1}$ (fev. 2006 a fev. 2008); (ii) de máximo crescimento ${ }^{2}$ e (iii) de mínimo crescimento ${ }^{3}$; e incremento médio em diâmetro do tronco/mês ${ }^{4}$, no período de máximo ${ }^{5}$ e mínimo ${ }^{6}$ crescimento.

Means followed by standard deviation and percentage of increment maximum(IAmáx) and minimum (IAmín) compared to the cumulative increase in the period (IA), trunk diameter increment during the period: (i) total1 (Feb/2006 to Feb / 2008), (ii) maximum crescimento ${ }^{2}$ and (iii) minimum ${ }^{3}$ growth; trunk diameter increment/month ${ }^{4}$ and in the maximum ${ }^{5}$ and minimum ${ }^{6}$ growth.

ao elevado teor de umidade do solo (Figura 3) e ao maior número de dias nublados afetando a respiração e a translocação de nutrientes pelas raízes e a fotossíntese, com reflexo no incremento do tronco. No mês subsequente (fevereiro de 2007), verificou-se, da mesma forma, menor incremento do tronco em razão do elevado teor de umidade remanescente no solo, mesmo com a redução da precipitação.

As árvores apresentaram curva de tendência do crescimento em diâmetro do tronco (Figura 2B) observada comumente em plantações florestais (MELLO, 1968; GONÇALVES etal., 1997; GAVA, 1997; ALMEIDA, 2009), com elevada taxa de incremento do tronco nos anos iniciais, seguida de estabilização, pelo aumento da demanda dos fatores de crescimento como água, nutrientes, luz etc.

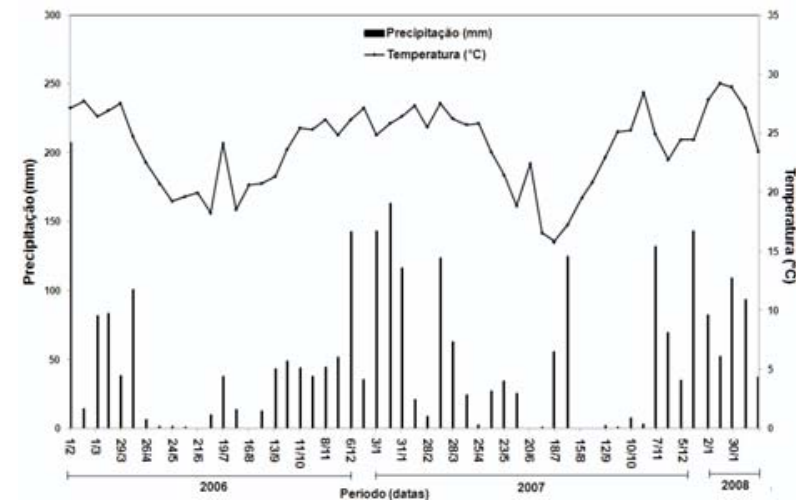

Figura 4-Precipitação e temperatura média da área experimental. Figure 4 - Precipitation and average temperature of the experimental area.
O incremento acumulado em diâmetro do tronco das árvores indicam, da mesma forma, os períodos de máximo e mínimo crescimento, mais evidente nos tratamentos 2 e 3 (Figura 5). Na seção transversal do lenho das árvores, verificou-se a formação de camadas de crescimento, perceptíveis macroscopicamente (Figura 5, seção A), sobretudo no período próximo aos 36 meses de idade.

As camadas de crescimento no lenho das árvores de eucalipto, além do componente genético, são formadas em resposta da atividade cambial à sazonalidade climática. Na estação de crescimento, o câmbio forma anéis de crescimento de lenho inicial, caracterizados por vasos de maior diâmetro e frequência (Figura 5, seção transversal microscópica do lenho A1) e lenho de menor densidade; na fase de estresse hídrico, os anéis de crescimento de lenho tardio formados são caracterizados por vasos de menor diâmetro e frequência (Figura 5, seção transversal microscópica do lenho A2) e lenho de maior densidade, conforme constatado por Leal et al. (2004) em árvores de Eucalyptus globulus, em Portugal; e por Tomazello Filho (2006) e Tomazello Filho et al. (2008) em árvores de Eucalyptus grandis x urophylla, no Brasil. Da mesma forma, Wimmmer et al. (2002ab) destacaram que o aumento do déficit de água no solo induz a formação de anéis de crescimento no lenho de Eucalyptus globulus e E. nitens, com vasos de menor diâmetro e frequência com aumento da densidade do lenho (lenho final). A densidade do lenho de eucalipto, segundo Downes et al. (1997; 2000), é resultado da sua estrutura anatômica: dimensões e frequência dos vasos e da espessura da parede celular das fibras, ao longo dos anéis de crescimento.

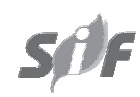

Revista Árvore, Viçosa-MG, v.34, n.6, p.979-990, 2010 


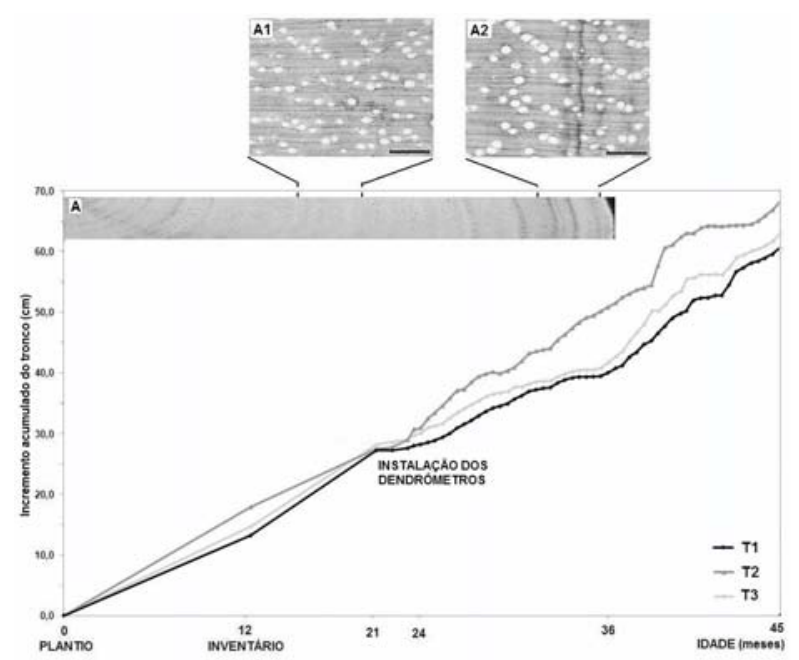

Figura 5 - Incremento médio acumulado do tronco das árvores de eucalipto nos três tratamentos, seção transversal macroscópica do lenho (Trat. 3 com 36 meses) e seções transversais microscópicas produzidas em período normal (A1) e com estresse hídrico (A2).

Figure 5 - Accumulated trunk increment of eucalyptus trees in the three treatments and macroscopic cross-section of wood (Trat. 3 with 36 month) and microscopic cross-sections produced when normal (A1) and drought (A2) period.

As correlações entre as variáveis climáticas e o incremento em diâmetro do tronco das árvores foram baixas (Tabela 5, antes da aplicação da defasagem), exceto para a temperatura máxima e para o déficit de pressão de vapor de água, com valores de $\mathrm{R}=0,32$ e $-0,46$, respectivamente, significativos a $5 \%$ de probabilidade. Da mesma forma, Wimmer et al. (2002a) e Drew et al. (2008) mostraram boas correlações entre a taxa de incremento em diâmetro do tronco de eucalipto e a temperatura.

As árvores, no entanto expressam o estímulo em crescimento do tronco em resposta às variáveis climáticas, após um intervalo de tempo considerado como período de defasagem (lag). Pela aplicação da autorregressão, determinou-se o período de defasagem entre o incremento em diâmetro do tronco e as variáveis climáticas, obtendo correlações maiores. O coeficiente de correlação (R) foi maior para todas as variáveis climáticas, com defasagem de 28 dias (Tabela 5, depois da aplicação da defasagem), destacando-se a precipitação, que passou de 0,10 para 0,51 e as temperaturas média e mínima, que passaram de 0,23 e 0,17 para 0,48 e 0,50, respectivamente, significativas a 5\% de probabilidade. Resultado similar foi obtido por Downes et al. (1999) em árvores de E. globulus e E. nitens com melhores correlações entre o incremento do seu tronco, após um período de defasagem das variáveis climáticas. Nesse sentido, Laclau et al. (2005) sugeriram que as árvores de eucalipto formam raízes finas e produzem fotossintetizados em resposta às variações climáticas, ativando o crescimento em diâmetro do tronco com defasagem de cerca de 28 dias. A correlação entre a umidade nas diferentes profundidades do solo (15$300 \mathrm{~cm}$ ) e o incremento em diâmetro do tronco foi baixa e não significativa, antes e depois da aplicação da defasagem, exceto para umidade na camada mais profunda do solo $(300 \mathrm{~cm})$, com melhora do valor do coeficiente e significativo após a aplicação da defasagem.

Das variáveis climáticas relacionadas para explicar o incremento em diâmetro do tronco das árvores (IC) analisadas em regressão Stepwise, a 5\% de probabilidade, selecionou-se o déficit de pressão de vapor de água (DPV) para compor o modelo de regressão, caracterizado

Tabela 5 - Coeficientes de correlação (R) entre as variáveis climáticas e o incremento do tronco das árvores de eucalipto antes e depois do período de defasagem (lag de 28 dias).

Table 5 - Correlation coefficients ( $R$ ) among the climatic variables and the trunk increment of eucalyptus trees before and after the delay (lag of 28 days).

\begin{tabular}{llr}
\hline Parâmetro & $\begin{array}{c}\text { Coeficiente de correlação (R) } \\
\text { antes }\end{array}$ & depois \\
\hline TEMP $_{\text {média }}$ & $0,23^{\text {ns }}$ & $0,48^{*}$ \\
TEMP $_{\text {máx. }}$ & $0,32^{*}$ & $0,46^{*}$ \\
TEMP $_{\text {mín. }}$ & $0,32^{*}$ & $0,32^{*}$ \\
Precipitaça $_{\text {Déficit de Pressão }}$ & $0,32^{*}$ & $0,32^{*}$ \\
de Vapor & $0,32^{*}$ & $0,32^{*}$ \\
$\mathrm{U}_{15}$ & $0,32^{*}$ & $0,32^{*}$ \\
$\mathrm{U}_{50}$ & $0,32^{*}$ & $0,32^{*}$ \\
$\mathrm{U}_{150}$ & $0,32^{*}$ & $0,32^{*}$ \\
$\mathrm{U}_{300}$ & $0,32^{*}$ & $0,32^{*}$ \\
\hline
\end{tabular}

${ }^{\text {ns }}$ não significativo a 5\% de probabilidade; * significativo a $5 \%$ de probabilidade.

$\mathrm{U}_{(0-15)}$ : umidade do solo na camada 0-15 cm; $\mathrm{U}_{(0-50)}$ : umidade do solo na camada $0-50 \mathrm{~cm} ; \mathrm{U}_{(0-150)}$ : umidade do solo na camada $0-150 \mathrm{~cm} ; \mathrm{U}_{(0-300)}$ : umidade do solo na camada $0-300 \mathrm{~cm}$ ${ }^{n s}$ not significant at $5 \%$ probability level, * significant at $5 \%$ probability.

${ }^{U}(0-15)$ : soil moisture in $0-15 \mathrm{~cm}$ layer, $U(0-50)$ : soil moisture in 0-50 cm layer, $U$ (0-150): soil moisture in the layer 0 $150 \mathrm{~cm}, U(0-300)$ : soil moisture in the layer $0-300 \mathrm{~cm}$. 
por IC $=5,0757-2,6738 * \mathrm{DPV}$, explicando $21 \%$ $\left(\mathrm{R}^{2}=0,21\right)$ da variação do total do incremento do tronco no período de 24 meses.

\subsection{Efeito da fertilização mineral}

Os tratamentos de fertilização mostraram maiores diferenças no incremento em diâmetro do tronco das árvores em fevereiro-maio/2006 e janeiro-março/2007 (Tabela 3). Nos demais meses e, principalmente no período de mínimo crescimento do tronco, o efeito dos tratamentos de fertilização indicou significativa redução (Figura 2A).

As árvores apresentaram taxas de incremento em diâmetro do tronco significativas e superiores no tratamento 2 (aplicação de K) em relação aos demais, principalmente, de fevereiro-maio/2006 e janeiro-março/ 2007 (Tabela 3; Figura 2A). O incremento acumulado do diâmetro do tronco das árvores (IA) foi de 3,08; 4,14; e de 3,28 cm nos tratamentos 1,2 e 3, respectivamente. Os parâmetros de incremento em diâmetro do tronco (Tabela 4) referentes ao período de máximo (IAmáx) e mínimo (IAmín) crescimento, incremento médio mensal (IMM) e no período de máximo (IMMmáx) e mínimo (IMMmín) crescimento foram superiores no tratamento 2 .

Os valores e variações do diâmetro e do volume do lenho do tronco nos períodos inicial (fevereiro/ 2006) e final (fevereiro/2008) indicaram que no tratamento 2 a variação (Ä) do diâmetro do tronco e do volume do lenho foram de 4,1 cm e 0,06 $\mathrm{m}^{3}$, superior e significativa estatisticamente em relação ao tratamento $1(3,5 \mathrm{~cm}$ e $\left.0,03 \mathrm{~m}^{3}\right)$ e não significativa em relação ao tratamento 3 (3,9 cm e 0,05 m³). O aumento do diâmetro do troncovolume do lenho das árvores, nos 24 meses de monitoramento, foi de 53-224, 50-187 e 52-186\% nos tratamentos 1, 2 e 3, respectivamente (Tabela 6).

O efeito positivo da adubação potássica no crescimento das árvores de eucalipto foi constatado por diversos autores, como Novais et al. (1986), Andrade et al. (1994), Gava (1997) e Faria et al. (2002), mostrando a importância do potássio para se atingir elevada produtividade das florestas em solos de Cerrado. Os inventários florestais realizados simultaneamente por Laclau et al. (2007), nos três tratamentos do experimento, demonstraram os mesmos resultados obtidos com o uso dos dendrômetros de aço.

As árvores do tratamento 3 (aplicação de $\mathrm{Na}$ ) apresentaram crescimento superior ao das árvores do tratamento 1 (controle), com incremento acumulado de 3,28 e 3,08 cm, respectivamente. O efeito positivo do Na no crescimento das árvores de eucalipto e na utilização com o K poderá diminuir os custos de implantação dos reflorestamentos. A substituição do potássio pelo sódio na fertilização do solo e em plantações de eucalipto foi mencionada por Judd et al. (1996) e Laclau et al. (2005), sendo de interesse prático e científico, pela utilização de fertilizantes mais baratos nas espécies vegetais tolerantes (MARSCHNER, 1995).

Tabela 6 - Diâmetro e volume do tronco das árvores de eucalipto no período inicial ( $22^{\circ}$ mês) e final ( $46^{\circ}$ mês) do experimento. Table 6 -Diameter and volume of eucalyptus trees at the initial $\left(22^{\text {th }}\right.$ month) and the final ( $46^{\text {th }}$ month) of the experiment.

\begin{tabular}{ccccc}
\hline Parâmetro & Periodo & \multicolumn{3}{c}{ Tratamento } \\
\cline { 3 - 5 } & & 1 & 2 & 3 \\
\hline Diâmetro do tronco & inicial & $6,6 \mathrm{a}(1,7)$ & $8,2 \mathrm{~b}(1,4)$ & $7,5 \mathrm{ab}(1,7)$ \\
$(\mathrm{cm})$ & final & $10,1 \mathrm{a}(2,6)$ & $12,3 \mathrm{~b}(2,5)$ & $11,4 \mathrm{ab}(2,8)$ \\
& $\Delta$ & 3,5 & 4,1 & 3,9 \\
& $\%$ & 53 & 50 & 52 \\
\hline Volume c/c & inicial & $0,0147 \mathrm{a}(0,00677)$ & $0,0301 \mathrm{~b}(0,0085)$ & $0,0243 \mathrm{~b}(0,0115)$ \\
$\left(\mathrm{m}^{2}\right)$ & final & $0,0476 \mathrm{a}(0,02555)$ & $0,0864 \mathrm{~b}(0,0373)$ & $0,0694 \mathrm{a}(0,0362)$ \\
& $\Delta$ & 0,0329 & 0,0563 & 0,0451 \\
& $\%$ & 224 & 187 & 186 \\
\hline
\end{tabular}

Médias seguidas de desvio-padrão; em uma mesma linha, médias seguidas de pelo menos uma letra igual não diferem significativamente ( $\mathrm{p}>0,05) ; \Delta$ - variação do diâmetro do tronco e volume das árvores do início ao final do período de monitoramento; e \% - porcentagem da variação $(\Delta)$ em relação ao diâmetro e volume inicial.

Means followed by standard deviation; in the same row, means followed by at least an equal letter do not differ significantly $(p>0.05) \Delta$-variation of stem diameter and tree volume from beginning to end of the monitoring period; \% - percentage of variation $(\Delta)$ compared to the initial diameter and volume. 


\section{CONCLUSÕES}

O crescimento em diâmetro do tronco de E. grandis foi afetado pelos tratamentos de fertilização mineral e pelas variáveis climáticas. As árvores que receberam aplicação de potássio apresentaram crescimento superior, com incremento acumulado no período total de 24 meses de 4,14 cm em relação às que receberam sódio e controle, com 3,28 e 3,08 cm, respectivamente. Os incrementos em diâmetro do tronco foram caracterizados por períodos de máximo e de mínimo crescimento, correspondentes a 55-59\% e 10-12\% do incremento total, respectivamente. As variáveis climáticas influenciaram as taxas de crescimento, com ênfase no déficit de pressão de vapor, sendo determinado um período de defasagem (lag) de 28 dias da resposta das árvores às variáveis climáticas.

\section{AGRADECIMENTOS}

À Fundação de Amparo à Pesquisa do Estado de São Paulo (FAPESP), pelo financiamento desta pesquisa; e à Estação Experimental de Itatinga/SP, do Departamento de Ciências Florestais da ESALQ/USP, pelo apoio.

\section{REFERÊNCIAS}

ALMEIDA, J. C. R. Nutrição, crescimento, eficiência do uso da água e de nutrientes em povoamentos de Eucalyptus grandis fertilizadas com potássio e sódio. 2009. 112f. Tese (Doutorado em Recursos Florestais) - Universidade de São Paulo, Piracicaba, 2009.

ANDRADE, A. M. et al. Efeitos da fertilização mineral e da calagem do solo na produção e na qualidade da madeira de eucalipto. Revista Árvore, v.18, n.1, p.69-78, 1994.

BOTOSSO, P. C.; TOMAZELLO FILHO, M. Aplicação de faixas dendrométricas na dendrocronologia: avaliação da taxa e do ritmo de crescimento do tronco de árvores tropicais e subtropicais. In: MAIA, N. B.; MARTOS, H. L.; BARRELLA, W. Indicadores ambientais: conceitos e aplicações. São Paulo: EDUC, 2001. p.145-171.

DÉTIENNE, P. et al. Rythmes de croissance de quelques essences de Guyane Français. Bois et Forêts dês Tropiques, v.217, n.2, p.63-76, 1988.
DELGADO, R. C. Avaliação de modelos físico-matemáticos para estimativa de umidade relativa do ar e déficit de pressão de vapor a partir de dados de temperatura do ar. 2007. 107f. Dissertação (Mestrado em Meteorologia Agrícola) Universidade Federal de Viçosa, Viçosa, MG, 2007.

DOWNES, G. et al. Sampling plantation eucalyptus: for wood and fibre properties. Melbourne: CSIRO, 1997. 132p.

DOWNES, G.; BEADLE, C.; WORLEDGE, D. Daily stem growth patterns in irrigated Eucalyptus globulus and Eucalyptus nitens in relation to climate. Trees, v.14, n.1, p.102-111, 1999.

DOWNES, G. M. et al. The commercial cambium: understanding the origin of wood property variation In: SAVIDGE, R. A. et al. Cell and molecular biology of wood formation. Oxford: BIOS Science, 2000. p.325-336.

DREW, D. M et al. Daily patterns of stem size variation in irrigated and non-irrigated Eucalyptus globulus. Tree Physiology, v.28, n.1, p.15731581, 2008.

FAHN, A. et al. Possible contributions of wood anatomy to the determination of the age of tropical trees. In: BORMANN, F. H.; BERLYN, G. Age and growth rate of tropical trees: new directions for research. New Haven: Yale University, 1980. p.31-54. (School of Forestry \& Environmental Studies, 94)

FARIA, G. E. et al. Produção e estado nutricional de povoamentos de Eucalyptus grandis, em segunda rotação, em resposta à adubação potássica.

Revista Árvore, v.26, n.5, p.577-584, 2002.

GAVA, J. L. Efeito de adubação potássica em plantios de Eucalyptus grandis conduzidos em segunda rotação em solos com diferentes teores de potássio trocável. Série Técnica IPEF, v.11, n.30, p.89-94, 1997.

GONÇALVES, J. L. M. et al. Soil and stand management for short-rotation plantations In: NAMBIAR, E. K. S.; BROWN, A. G. (Eds.). Management of soil, water, and nutrients in tropical plantation forests. Canberra: 1997. p.379-418. (Aciar, Monograph, 43). 
GREEN, J. W. Continuous measurements of radial variation in Eucalyptus pauciflora Sieb. Ex. Spreng. Australian Journal of Botany, v.17, n.2, p.191-198, 1969.

HUSCH, B.; MILLER, C. I.; BEERS, T. W. Forest mensuration. New York: Ronald, 1982. 401p

JUDD, T. S.; ATTIWWIL, P. M.; ADANS, A. M. Nutrient concentration in eucalyptus: a synthesis in relation to differences between tax, sites and components. In: ATTIWILL, P. M.; ADAMS, M. A. Nutrition of Eucalyptus. Melbourne: CSIRO, 1996. p.123-153.

LAMPRECHT, H. Silvicultura nos trópicos. Eschborn: GTZ, 1990. 343p.

LACLAU, J. P. et al. Nutrient cycling in a clonal stand of eucalyptus and an adjacent savana ecosystem in Congo: chemical composition of rainfall, trough fall and stem flow solutions.

Forest Ecology and Management, v.176, n.1/3, p.105-119, 2005.

LACLAU, J. P. et al. Processos de transferência e balanço de água e de nutrientes em povoamentos de Eucalyptus sp que receberam aplicações de nitrogênio e de biossólido: reflexos sobre a sustentabilidade. São Paulo: 2007. 65p. (Relatório apresentado a Fundação de Amparo à Pesquisa do Estado de São Paulo FAPESP).

LEAL, S. et al. Clonal and site variation of vessels in 7 year old Eucalyptus globulus. IAWA Journal, v.24, n.2, p.185-195, 2004.

MARIAUX, A. Les cernes dans les bois tropicaux Africains, nature et périodicité.. Bois et Forêts dês Tropiques, v.113, n.1, p. 23-37, 1967.

MARIAUX, A. La périodicité des cernes dans les bois de limba. Bois et Forêts dês Tropiques, v.128, n.1, p.39-53, 1969.

MARIAUX, A. La périodicité de formation des cernes dans le bois de I'Oukoume. Bois et Forêts dês Tropiques, v.131, n.1, p.37-50, 1970.
MARIEN, J. N.; THIBOUT, H. Les eucalyptus en France: rythmes de croissance en fonction des conditions climatiques. Recherches Sylvicoles. Paris, p. 273-299. 1980.

MARSCHNER, H. Mineral nutrition of higher plants. London: Academic Pres, 1995. 889p.

MELLO, H. A. Aspectos do emprego de fertilizantes minerais no reflorestamento de solos de cerrado do estado de São Paulo com Eucalyptus saligna. 1968. 176f. Tese (Doutorado em Silvicultura) - Escola Superior de Agricultura “Luiz de Queiroz”, Piracicaba, 1968.

NOVAIS, R. F.; BARROS, N. F.; NEVES, J. C. L. Interpretação de análise química do solo para o crescimento e desenvolvimento do Eucalyptus spp.: níveis críticos de implantação e manutenção. Revista Árvore, v.10, n.1, p.105-111, 1986.

POOLE, D. J. Diameter growth of 4-7 year old Eucalyptus regnans. New Zealand Forestry, v. 31, n.1, p.23-24, 1986.

SILVEIRA, R. L. V. A.; MALAVOLTA, E. Produção e características químicas da madeira juvenil de progênies de Eucalyptus grandis em função das doses de potássio na solução nutritiva. Scientia Forestalis, n.63, p.115-135, 2003.

TOMAZELLO FILHO, M. Efeito da irrigação e fertilização nas propriedades do lenho de árvores de Eucalyptus grandis $x$ urophylla. 2006. 135f. Tese (Livre-Docência) Escola Superior de Agricultura Luiz de Queiroz, Piracicaba, 2006.

TOMAZELLO FILHO, M. et al. Aplication of Xray techique in nondestructive evaluation of eucalypt wood. Maderas: Ciencia y Tecnologia, v.10, n.2, p.139-149, 2008.

VALENZIANO, S.; SCARAMUZZI, G. Preliminary observations on the seasonal diameter growth of Eucalyptus camaldulensis and E. viminalis. In: WORLD SYMPOSIUM ON MAN-MADE FORESTS AND THEIR INDUSTRIAL IMPORTANCE, 1967, Canberra. Anais... Canberra: Wood and Agriculture Organization of the United Nations, 1967. p.1923-1933.

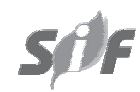

Revista Árvore, Viçosa-MG, v.34, n.6, p.979-990, 2010 
WIMMER, R.; DOWNES G. M.; EVANS, R.

Temporal variation of microfibril angle in Eucalyptus nitens grown in different irrigation regimes. Tree Physiology, v.22, n.7, p. 449-457, 2002.
WIMMER, R. High-resolution analysis of radial growth and wood density in Eucalyptus nitens, grown under different irrigation regimes.

Annales Forest Sciences, v.59, n.5-6, p.519524, 2002. 